\title{
Dihedral multi-reference alignment
}

\author{
Tamir Bendory, Dan Edidin, William Leeb, and Nir Sharon
}

January 5, 2022

\begin{abstract}
We study the dihedral multi-reference alignment problem of estimating the orbit of a signal from multiple noisy observations of the signal, acted on by random elements of the dihedral group. We show that if the group elements are drawn from a generic distribution, the orbit of a generic signal is uniquely determined from the second moment of the observations. This implies that the optimal estimation rate in the high noise regime is proportional to the square of the variance of the noise. This is the first result of this type for multi-reference alignment over a non-abelian group with a non-uniform distribution of group elements. Based on tools from invariant theory and algebraic geometry, we also delineate conditions for unique orbit recovery for multi-reference alignment models over finite groups (namely, when the dihedral group is replaced by a general finite group) when the group elements are drawn from a generic distribution. Finally, we design and study numerically three computational frameworks for estimating the signal based on group synchronization, expectation-maximization, and the method of moments.
\end{abstract}

\section{Introduction}

We study the dihedral multi-reference alignment (MRA) model

$$
y=g \cdot x+\varepsilon, \quad g \sim \rho, \quad \varepsilon \sim \mathcal{N}\left(0, \sigma^{2} I\right),
$$

where

- $x \in \mathbb{R}^{L}$ is a fixed (deterministic) signal to be estimated;

- $\rho$ is an unknown distribution defined over the simplex $\Delta_{2 L}$;

- $g$ is a random element of the dihedral group $D_{2 L}$, drawn i.i.d. from $\rho$, and acting on the signal by circular translation and reflection (see Figure 1);

- $\varepsilon$ is a normal isotropic i.i.d. noise with zero mean and variance $\sigma^{2}$.

We wish to estimate the signal $x$ from $n$ realizations (observations) of $y$,

$$
y_{i}=g_{i} \cdot x+\varepsilon_{i}, \quad i=1, \ldots, n,
$$


while the corresponding group elements $g_{1}, \ldots, g_{n}$ are unknown. We note, however, that the signal can be identified only up to the action of an arbitrary element of the dihedral group. Therefore, unless a prior information on the signal is available, the goal is estimating the orbit of signals $\left\{g \cdot x \mid g \in D_{2 L}\right\}$. This type of problem is often dubbed an orbit recovery problem.

The model (1.1) is an instance of the more general MRA problem that was studied thoroughly in recent years $[9,14,8,3,2,21,4,39,36,11,10,43,1,32,6,35,27,30,28,22,17,19]$. In its generalized version, the MRA model is formulated as (1.1), but the signal $x$ may lie in an arbitrary vector space (not necessarily $\mathbb{R}^{L}$ ), the dihedral group $D_{2 L}$ is replaced by an arbitrary group $G$, and $g \sim \rho$ is a distribution over $G$ (in some cases, an additional fixed linear operator acting on the signal is also considered, e.g., $[10,8,18,12])$. The goal is to estimate the orbit of $x$, under the action of the group $G$.

Most of the previous studies on MRA have considered the uniform (or Haar) distribution $\rho$ over the group elements. In particular, it was shown that in many cases, such as $x \in \mathbb{R}^{L}$ and a uniform distribution over $\mathbb{Z}_{L}$, the third moment suffices to recover a generic signal uniquely, and consequently $n / \sigma^{6} \rightarrow \infty$ is a necessary condition for accurate estimation of generic signal $[8,34,39,14]$. In fact, this follows from a general result that in the low SNR regime $\sigma \rightarrow \infty$ (with a fixed dimension $L$ ), a necessary condition for signal identification is $n / \sigma^{2 d} \rightarrow \infty$, where $d$ is the lowest order moment that identifies the orbit of signals uniquely [8, 4, 11, 39] (see [43] for sample complexity analysis in high dimensions).

The effect of non-uniform distribution on the sample complexity was first studied in [2] for the abelian group $\mathbb{Z}_{L}$ and $x \in \mathbb{R}^{L}$. It was shown that in this case the second moment suffices to identify the orbit of generic signals uniquely for almost any non-uniform distribution (rather than the third moment if the distribution is uniform). In this work, we extend [2] for the non-abelian group $D_{2 L}$ and show that for a generic distribution and signal, the second moment identifies the orbit of solutions. This implies that a necessary condition for accurate orbit recovery under the model (1.1) for $\sigma \rightarrow \infty$ and fixed $L$ is $n / \sigma^{4} \rightarrow \infty$. This is the first result of this type for multireference alignment over a non-abelian group with a non-uniform distribution of group elements. The fact that the group $D_{2 L}$ is non-abelian makes the analysis of the orbit recovery problem significantly more difficult. The reason is that this action of the dihedral group on $\mathbb{R}^{N}$ cannot be diagonalized as we explain in Remark 2.1. It follows that there is no basis where the entries of the moment tensors are monomials. By contrast, a previous work for the cyclic group $\mathbb{Z}_{L}$ took advantage of the fact that the entries of the moment tensors are monomials when expressed in the Fourier basis [2]. The main theoretical results are summarized as follows.

Theorem 1.1 (informal statement of the main theorem). Consider the dihedral MRA problem (1.1) with a generic probability distribution $\rho$. Then, the first and second order moments of $y$ are sufficient to uniquely identify almost all orbits.

Corollary 1.2 (sample complexity). Consider the dihedral MRA problem (1.1) in the low SNR regime $\sigma \rightarrow \infty$. For a generic probability distribution and a generic signal, $n / \sigma^{4} \rightarrow \infty$ is a necessary condition for accurate orbit identification.

Theorem 1.1 is formulated in technical terms in Theorem 2.3, which is proved in Section 2.2. The proof is based on algebraic geometry tools and is not constructive, namely, it does not provide an explicit algorithm of how to recover the signal from the first and second moment. Section 2.2 also discusses the precise meaning of the notion of generic signal and distribution. In Section 2.3, 


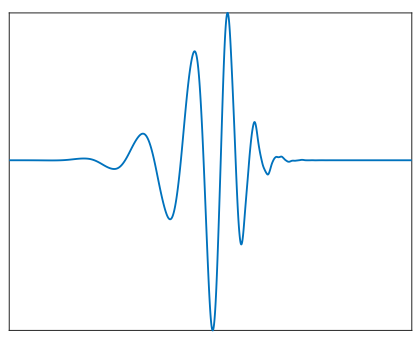

(a) Signal

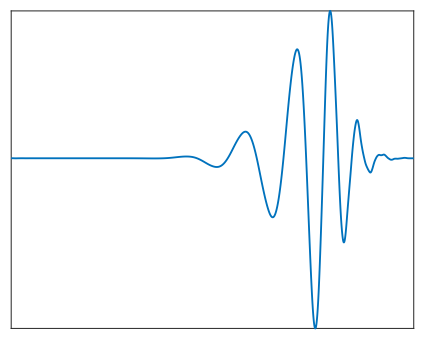

(b) Shifted signal

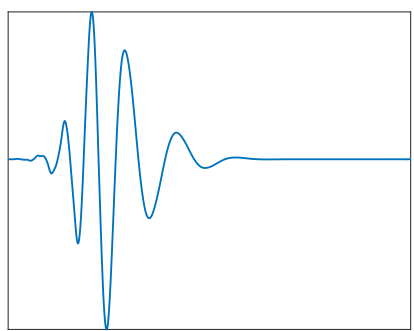

(c) Shifted and reflected signal

Figure 1: An example of the action of the dihedral group. The MRA problem (1.1) entails estimating a signal, up to a global circular shift and reflection, from multiple noisy copies of the signal acted upon by random elements of the dihedral group.

we use invariant theory to delineate general conditions for orbit recovery from the second moment in general MRA models over finite groups.

The MRA model is mainly motivated by the molecular structure reconstruction problem in single-particle cryo-electron microscopy (cryo-EM) [12]. The aim of a cryo-EM experiment is constituting a 3-D molecular structure from multiple observations. In each observation, the 3-D structure is acted upon by a random element of the non-abelian group of 3-D rotations $\mathrm{SO}(3)$. In addition, the distribution over $\mathrm{SO}(3)$ is usually non-uniform and unknown [51, 37, 7, 47]. Therefore, this paper is an important step towards understanding the statistical properties and sample complexity of the cryo-EM problem

Section 3 introduces three statistical estimation frameworks to recover the orbit of $x$. The first framework is based on estimating the missing group elements using the method of group synchronization $[49,10]$. Once the group elements were accurately estimated, estimating the signal can be obtained by aligning the observations and averaging out the noise. However, reliable estimation of group elements is possible only if the noise level is low enough. To estimate the signal in high noise levels, we also suggest maximizing the marginalized maximum likelihood using expectation-maximization (EM). The EM algorithm provides accurate estimations in a wide range of SNR regimes, although we have no theoretical guarantees to support it. Unfortunately, the computational burden of EM rapidly increases with the number of observations $n$ and the noise level. As a third method, we propose an estimator based on the method of moments, which works quite well in all SNRs and whose computational burden is roughly constant with the noise level and moderately increases with $n$. According to Theorem 1.1, we only use the first and second moments for the estimation. As with EM, characterizing the properties of the method of moments is left for future research; see further discussion in Section 3.

\section{Theory}

\subsection{The dihedral group}

The dihedral group $D_{2 L}$ is a group of order $2 L$, which is usually defined as the group of symmetries of a regular $L$-gon in $\mathbb{R}^{2}$. It is generated by a rotation $r$ of order $L$ corresponding to rotation by 
an angle $2 \pi / L$ and a reflection $s$ of order 2 . Since rotation does not commute with reflection, the group $D_{2 L}$ is not abelian, but the relation $r s=s r^{-1}$ holds instead. Since $r$ has order $L, r^{-1}=r^{L-1}$. The elements of $D_{2 L}$ can be enumerated as

$$
\left\{1, r, \ldots, r^{L-1}, s, r s, \ldots, r^{L-1} s\right\}
$$

where 1 is the identity element. Note that the subset $\left\{1, r, \ldots, r^{L-1}\right\}$ is a normal $\operatorname{subgroup}^{1}$ isomorphic to the cyclic group $\mathbb{Z}_{L}$. MRA over the group $\mathbb{Z}_{L}$ was studied thoroughly, see for example $[9,14,2]$.

There are two natural ways to describe the action of the dihedral group, $D_{2 L}$, on $\mathbb{R}^{L}$ one in the time (or spatial) domain and one in the Fourier (frequency) domain. Explicitly, in the time domain, the action of the dihedral group on a signal $x \in \mathbb{R}^{L}$ is given by

$$
\begin{aligned}
& (r \cdot x)[\ell]=x[(\ell-1) \bmod L], \\
& (s \cdot x)[\ell]=x[-\ell \bmod L] .
\end{aligned}
$$

Namely, $r$ cyclically shifts a signal by one entry, and $s$ reflects the signal. The action of the dihedral group is illustrated in Figure 1.

If we apply the discrete Fourier transform to $\mathbb{R}^{L}$, then we can identify $\mathbb{R}^{L}$ with the real subspace of $\mathbb{C}^{L}$ consisting of $L$-tuples $(\hat{x}[0], \ldots, \hat{x}[L-1]) \in \mathbb{C}^{L}$ satisfying the condition $\overline{\hat{x}[\ell]}=\hat{x}[-\ell \bmod L]$, where $\overline{\hat{x}[\ell]}$ is the conjugate of $\hat{x}[\ell]$. In this case, the action of $D_{2 L}$ is given by:

$$
\begin{aligned}
(r \cdot \hat{x})[\ell] & =e^{2 \pi \iota \ell / L} \hat{x}[\ell], \\
(s \cdot \hat{x})[\ell] & =\overline{\hat{x}[\ell]}=\hat{x}[-\ell \bmod L] .
\end{aligned}
$$

Remark 2.1. We can see from this description that the action of $D_{2 L}$ cannot be diagonalized for the following reason. If the action could be diagonalized, then there would have to be a basis for $\mathbb{R}^{L}$ which consists of simultaneous eigenvectors of the rotation $r$ and the reflection s. However, the only eigenvector of the rotation $r$ which is also invariant under the action of the reflection $s$ is the vector $(1,0, \ldots, 0)$. For a further reference, see [46, p. 37].

\subsection{Unique orbit recovery in dihedral MRA}

We are now ready to present and prove the main result of this paper. Let $\rho \in \Delta_{2 L}$ be a probability distribution on $D_{2 L}$. We denote the probability of $r^{k}$ by $p[k]$ and the probability of $r^{k} s$ by $q[k]$. Let $p$ and $q$ represent the vectors $(p[0], \ldots, p[L-1])$ and $(q[0], \ldots, q[L-1])$, respectively. Let $C_{z} \in \mathbb{R}^{L \times L}$ be a circulant matrix generated by $z \in \mathbb{R}^{L}$, namely, the $i$-th column of $C_{z}$ is given by $z[(i-\ell) \bmod L]$ for $\ell=0, \ldots, L-1$. Let $D_{z} \in \mathbb{R}^{L \times L}$ be a diagonal matrix whose entries are $z$. A direct calculation shows that the first two moments of the observations of (1.1) are given by the following expressions (compare with [2]).

Lemma 2.2. Consider the dihedral MRA model (1.1). The first moment of $y, M^{1} \in \mathbb{R}^{L}$, is given by

$$
\mathbb{E} y:=M^{1}(x, \rho)=C_{x} p+C_{s x} q=C_{p} x+C_{q} s x .
$$

The second moment of $y, M^{2} \in \mathbb{R}^{L \times L}$, is given by

$$
\mathbb{E} y y^{T}:=M^{2}(x, \rho)=C_{x} D_{p} C_{x}^{T}+C_{s x} D_{q} C_{s x}^{T}+\sigma^{2} I .
$$

\footnotetext{
${ }^{1}$ A subgroup $H<G$ is normal if it is invariant under conjugation by elements of $G$.
} 
Hereafter, we assume that the noise variance $\sigma^{2}$ is known, and thus the bias term $\sigma^{2} I$ can be removed. Indeed, the variance of the average of each observation $\frac{1}{\sqrt{L}} \sum_{\ell=0}^{L-1} y_{i}[\ell]$ (which is invariant under the group action) is an unbiased estimator of the noise variance, and is consistent as $\sigma^{4} / n \rightarrow 0$. We also remark that in many applications, including cryo-EM, the noise level can often be readily estimated from the data [12].

To present the main result of this paper, it will be convenient to consider the Fourier counterpart of the moments, defined by

$$
\begin{aligned}
& \hat{M}^{1}=\mathbb{E} F y=F M^{1}(x, \rho), \\
& \hat{M}^{2}=\mathbb{E} F y(F y)^{*}=F M^{2}(x, \rho) F^{*},
\end{aligned}
$$

where $F \in \mathbb{C}^{L \times L}$ is the discrete Fourier transform (DFT) matrix.

We say that a condition holds for generic signals (or distributions) if the set of signals (distributions) for which the condition does not hold is defined by polynomial conditions. The precise meaning of generic signals, in the context of this work, is discussed at the end of this section.

The main result of this paper is as follows.

Theorem 2.3 (Orbit recovery). For generic signal $x$ and generic distribution $\rho$, the $D_{2 L}$ orbit of $x$ is uniquely determined by $\hat{M}^{1}[0]=\hat{x}[0]$ and at most $\sim 2.5 L$ entries of the matrix $\hat{M}^{2}$. More precisely, there exist non-zero polynomials $Q_{1}, \ldots, Q_{r}$ such that if $Q_{1}(x, \rho), \ldots, Q_{r}(x, \rho)$ are not all zero, then for any $\left(z, \rho^{\prime}\right)$ with $M^{1}\left(z, \rho^{\prime}\right)=M^{1}(x, \rho)$ and $M^{2}\left(z, \rho^{\prime}\right)=M^{2}(x, \rho), z$ is in the same $D_{2 L}$ orbit as $x$.

Remark 2.4. Our method of proof necessarily requires that all of the entries of Fx are non-zero, where $F$ is the discrete Fourier transform matrix; similar assumptions are often stated in the MRA literature, see for example [14, 3, 39, 11]. However, our proof also requires that additional, less explicit, polynomials in the entries of $x, \rho$ be non-vanishing. This is discussed at the end of the proof.

Proof. Let us define

$$
\begin{aligned}
& \hat{p}=(\hat{p}[0], \ldots, \hat{p}[L-1]), \\
& \hat{q}=(\hat{q}[0], \ldots, \hat{q}[L-1]), \\
& \hat{x}=(\hat{x}[0], \ldots, \hat{x}[L-1]) .
\end{aligned}
$$

Note that the second moment in Fourier domain can be written as

$$
\hat{M}^{2}=\frac{1}{L}\left(D_{\hat{x}} C_{\hat{p}} D_{\bar{x}}+D_{\widehat{x}} C_{\hat{q}} D_{\hat{x}}\right) .
$$

Moreover, since $p, q, x$ are real, we have the symmetry relations

$$
\begin{aligned}
\hat{x}[L-i] & =\overline{x[i]}, \\
\hat{p}[L-i] & =\overline{\hat{p}[i]}, \\
\hat{q}[L-i] & =\overline{\hat{q}[i]} .
\end{aligned}
$$

Define

$$
M_{i, j}=\hat{p}[i+j] \hat{x}[i] \hat{x}[j]+\hat{q}[L-i-j] \hat{x}[L-i] \hat{x}[L-j]
$$


so $M_{i, j}$ is $L \hat{M}^{2}[i, L-j]$. Our goal is to show that knowledge of $\hat{M}^{1}[0]$ and $O(L)$ of the entries $M_{i, j}$ determine the orbit of $x$.

Since $\rho$ is a probability distribution, we note that

$$
\hat{p}[0]+\hat{q}[0]=p[0]+\ldots+p[L-1]+q[0]+\ldots+q[L-1]=1 .
$$

Thus, $M_{i,-i}=|\hat{x}[i]|^{2}$. It follows that knowledge of $M^{2}(x, \rho)$ determines the power spectrum of $x$. Replacing $x$ by the vector whose Fourier transform has entries $\hat{x}[i] /|\hat{x}[i]|$, we may assume that each $\hat{x}[i]$ lies on the unit circle. Since $\hat{x}[0]$ is real, we take $\hat{x}[0]=1$. With this assumption, the formula for $M_{i, j}$ can be written as

$$
M_{i, j}=\hat{p}[i+j] \hat{x}[i] \hat{x}[j]+\hat{q}[L-i-j] /(\hat{x}[i] \hat{x}[j]) .
$$

Given a vector $x$ and distribution $\rho$, consider the set $I$ of vectors $z \in \mathbb{R}^{L}$ such that $M^{1}\left(z, \rho^{\prime}\right)=$ $M^{1}(x, \rho)$ and $M^{2}\left(z, \rho^{\prime}\right)=M^{2}(x, \rho)$ for some probability distribution $\rho^{\prime}$ on the dihedral group $D_{2 L}$. We will show that for generic $(x, \rho)$ there are only $2 L$ possible $z$ 's in this set. Note that the distribution is uniquely determined by the signal $z$, because the moments are linear functions of the distribution. Since the $D_{2 L}$ orbit of $x$ is contained in the set $I$, we conclude that the orbit of $x$ is determined by the moments of degree one and two.

Determining that the set $I$ consists of at most $2 L$ vectors is equivalent to showing that the following system of equations has at most $2 L$ solutions:

$$
\hat{p}^{\prime}[i+j] \hat{z}[i] \hat{z}[j]+\hat{q}^{\prime}[L-i-j] /(\hat{z}[i] \hat{z}[j])=M_{i, j}
$$

where $|\hat{z}[i]|=1, \hat{z}$ is the Fourier transform of a vector in $\mathbb{R}^{L}$, and $\hat{\rho}^{\prime}=\left(\hat{p}^{\prime}, \hat{q}^{\prime}\right)$ is the Fourier transform of a probability distribution on $D_{2 L}$. Consider the equations

$$
\hat{p}^{\prime}[1] \hat{z}[\ell] \hat{z}[1-\ell]+\hat{q}^{\prime}[L-1] /(\hat{z}[\ell] \hat{z}[1-\ell])=M_{\ell, 1-\ell}, \quad \ell=0, \ldots, L-1,
$$

where the indices are taken modulo $L$. For each fixed $\ell$, we can view equation (2.10) as a linear equation in $\hat{p}^{\prime}[1], \hat{q}^{\prime}[L-1]$. For the system to have a solution, it must be consistent. Taking the pair of equations when $\ell=1$ and $\ell=m+1$ with $m \geq 1$, we obtain

$$
\hat{q}^{\prime}[L-1]=\frac{\left(\hat{z}[1] \hat{z}[m+1]^{2} M_{1,0}-\hat{z}[1]^{2} \hat{z}[m] \hat{z}[m+1] M_{m+1,-m}\right)}{\hat{z}[m+1]^{2}-\hat{z}[1]^{2} \hat{z}[m]^{2}} .
$$

Equating equation (2.11) with $m=1$ and $m=n+1$, we see that $\hat{z}[n+1]$ satisfies the following quadratic equation in terms of $\hat{z}[1], \hat{z}[2], \hat{z}[n]$ :

$$
\begin{array}{r}
\left(M_{2,-1}^{2} \hat{z}[1] \hat{z}[2]-M_{1,0} \hat{z}[1]^{3}\right) \hat{z}[n+1]^{2}+M_{n+1,-n}^{2}\left(\hat{z}[1]^{4} \hat{z}[n]-\hat{z}[2]^{2} \hat{z}[n]\right) \hat{z}[n+1]+ \\
M_{1,0}^{2} \hat{z}[1] \hat{z}^{2}[2] \hat{z}[n]^{2}-M_{2,-1}^{2} \hat{z}[1]^{3} \hat{z}[2] \hat{z}^{2}[n]=0 .
\end{array}
$$

Note that expressions of the form $M_{i, j}^{2}$ refer to exponents in this formula.

If $n>2$, the three equations from $(2.9)$ with $(i, j)=(n+1,0),(n, 1),(n-1,2)$, respectively, yield three linear equations for $\hat{p}^{\prime}[n+1], \hat{q}^{\prime}[L-n-1]$ whose coefficients are rational expressions in $\hat{z}[1], \hat{z}[2], \hat{z}[n-1], \hat{z}[n], \hat{z}[n+1]$. The same analysis as above shows that $\hat{z}[n+1]$ satisfies an additional quadratic equation in $\hat{z}[1], \hat{z}[2], \hat{z}[n-1], \hat{z}[n]$ :

$$
\begin{aligned}
& \left(M_{n, 1}^{2} \hat{z}[1] \hat{z}[n]-M_{n-1,2}^{2} \hat{z}[2] \hat{z}[n-1]\right) \hat{z}[n+1]^{2}+M_{n+1,0}\left(\hat{z}[2]^{2} \hat{z}[n-1]^{2}-\hat{z}[1]^{2} \hat{z}[n]^{2}\right) \hat{z}[n+1] \\
& +\left(M_{n-1,2}^{2} \hat{z}[1]^{2} \hat{z}[2] \hat{z}[n-1] \hat{z}[n]^{2}-M_{n, 1}^{2} \hat{z}[1] \hat{z}[2]^{2} \hat{z}[n-1]^{2} \hat{z}[n]\right)=0 .
\end{aligned}
$$


Since $\hat{z}[n+1]$ satisfies the two non-equivalent quadratic equations (2.12) and (2.13), we can solve for $\hat{z}[n+1]$ in terms of $\hat{z}[1], \hat{z}[2]$ and $\hat{z}[n]$ and we obtain the following expression for $\hat{z}[n+1]$ as a rational function of $\hat{z}[1], \hat{z}[2], \hat{z}[n-1], \hat{z}[n]$ :

$$
\hat{z}[n+1]=\frac{a}{b}
$$

where

$$
\begin{aligned}
a & =M_{1,0}^{2} M_{n, 1}^{2}\left(\hat{z}[1]^{2} \hat{z}[2]^{2} \hat{z}[n]^{3}-\hat{z}[1]^{4} \hat{z}[2]^{2} \hat{z}[n-1]^{2} \hat{z}_{n}\right)+M_{2,-1}^{2} M_{n, 1}^{2}\left(\hat{z}[1]^{2} \hat{z}[2]^{3} \hat{z}[n-1]^{2} \hat{z}[n]-\hat{z}[1]^{4} \hat{z}[2] \hat{z}[n]^{3}\right) \\
& +M_{1,0}^{2} M_{n-1,2}^{2}\left(\hat{z}[1]^{5} \hat{z}[2] \hat{z}[n-1] \hat{z}[n]^{2}-\hat{z}[1] \hat{z}[2]^{3} \hat{z}[n-1] \hat{z}[n]^{2}\right),
\end{aligned}
$$

and

$$
\begin{aligned}
b & =M_{n-1,1} M_{n+1,-n}\left(\hat{z}[1] \hat{z}[2]^{2} \hat{z}[n]^{2}-\hat{z}[1]^{5} \hat{z}[n]^{2}\right)+M_{n-1,2}^{2} M_{n+1,-n}^{2}\left(\hat{z}[1]^{4} \hat{z}[2] \hat{z}[n-1] \hat{z}[n]-\hat{z}[2]^{3} \hat{z}[n-1] \hat{z}[n]\right) \\
& +M_{n+1,0}^{2} M_{2,-1}^{2}\left(\hat{z}[1] \hat{z}[2]^{3} \hat{z}[n-1]^{2}-\hat{z}[1]^{3} \hat{z}[2] \hat{z}[n]^{2}\right)+M_{n+1,0}^{2} M_{1,0}^{2}\left(\hat{z}[1]^{5} \hat{z}[n]^{2}-\hat{z}[1]^{3} \hat{z}[2]^{2} \hat{z}[n-1]^{2}\right) .
\end{aligned}
$$

When $n=2$, the equations in (2.9) corresponding to $(i, j)=(2,1)$ and $(i, j)=(1,2)$ are identical so we need another method to express $\hat{z}[3]$ as a rational function of $\hat{z}[1], \hat{z}[2]$. To get a second quadratic equation in this case, consider the equations of (2.9) corresponding to the pairs $(2,0),(1,1),(3,-1)$ to obtain the quadratic equation

$$
\left(M_{1,1}^{2} \hat{z}[1]^{2}-M_{2,0}^{2} \hat{z}[2]\right) \hat{z}[3]^{2}+M_{3,-1}^{2} \hat{z}[1]\left(\hat{z}[2]^{2}-\hat{z}[1]^{4}\right)+\hat{z}[1]^{4} \hat{z}[2]\left(M_{2,0}^{2} \hat{z}[1]^{2}-M_{1,1}^{2} \hat{z}[2]\right)=0 .
$$

We then obtain the following expression for $\hat{z}[3]$ as a rational function of $\hat{z}[1]$ and $\hat{z}[2]$ :

$$
\frac{\hat{z}[1] \hat{z}[2]\left(M_{1,0}^{2} M_{2,0}^{2} \hat{z}[1]^{4}-M_{1,0} M_{1,1}^{2} \hat{z}[1]^{2} \hat{z}[2]-M_{2,0} M_{2,-1} \hat{z}[1]^{2} \hat{z}[2]+M_{1,0} M_{2,0} \hat{z}[2]^{2}\right)}{\left(M_{1,0} M_{3,-1} \hat{z}[1]^{4}-M_{2,-1} M_{3,-1} \hat{z}[1]^{2} \hat{z}[2]-M_{1,1} M_{3,-2} \hat{z}[1]^{2} \hat{z}[2]+M_{2,0} M_{3,-2} \hat{z}[2]^{2}\right)} .
$$

At this point we have shown that knowledge of $\hat{z}[1], \hat{z}[2]$ determine $\hat{z}[n+1]$ for $n \geq 2$, assuming that the rational expressions (2.14) and (2.18) are well defined (see the discussion at the end of the proof). We can also use the quadratic equations (2.12) (with $n=3$ ) and (2.17) to obtain a second expression for $\hat{z}[3]^{2}$ as a rational function of $\hat{z}[1]$ and $\hat{z}[2]$. Equating this expression for $\hat{z}[3]^{2}$ with the square of the expression for $\hat{z}[3]$ given by (2.18), we obtain the following palindromic quartic equation for $\hat{z}[2]$ in terms of $\hat{z}[1]$ :

$$
A_{0} \hat{z}[1]^{8}+A_{1} \hat{z}[1]^{6} \hat{z}[2]+A_{2} \hat{z}[1]^{4} \hat{z}[2]^{2}+A_{1} \hat{z}[1]^{2} \hat{z}[2]^{3}+A_{0} \hat{z}[1]^{4}=0
$$

where

$$
\begin{aligned}
A_{0}= & M_{1,0}^{2} M_{2,0}^{2}-M_{1,0} M_{2,0} M_{3,-2} M_{3,-1} \\
A_{1}= & -2 M_{1,0}^{2} M_{1,1} M_{2,0}-2 M_{1,0} M_{2,-1} M_{2,0}^{2}+M_{1,1} M_{2,0} M_{3,-2}^{2} \\
& +M_{1,0} M_{1,1} M_{3,-2} M_{3,-1}+M_{2,-1} M_{2,0} M_{3,-2} M_{3,-1}+M_{1,0} M_{2,-1} M_{3,-1}^{2} \\
A_{2}= & M_{1,0}^{2} M_{1,1}^{2}+2 M_{1,0} M_{1,1} M_{2,-1} M_{2,0}+2 M_{1,0}^{2} M_{2,0}^{2}+M_{2,-1}^{2} M_{2,0}^{2} \\
& -M_{1,1}^{2} M_{3,-2}^{2}-M_{2,0}^{2} M_{3,-2}^{2}-2 M_{1,1} M_{2,-1} M_{3,-2} M_{3,-1}-M_{1,0}^{2} M_{3,-1}^{2}-M_{2,-1}^{2} M_{3,-1}^{2} .
\end{aligned}
$$


Taking the complex conjugate of (2.19) and using the fact that $\hat{z}[i]$ lies on the unit circle so $\overline{\hat{z}[i]}=\hat{z}[i]^{-1}$, we obtain

$$
\overline{A_{0}} \hat{z}[1]^{-8}+\overline{A_{1}} \hat{z}[1]^{-6} \hat{z}[2]^{-1}+\overline{A_{2}} \hat{z}[1]^{-4} \hat{z}[2]^{-2}+\overline{A_{1}} \hat{z}[1]^{-2} \hat{z}[2]^{-3}+\overline{A_{0}} \hat{z}[1]^{-4}=0 .
$$

Multiplying (2.20) by $\hat{z}[1]^{8} \hat{z}[2]^{4}$, we obtain a second quartic equation satisfied by $\hat{z}[2]$ :

$$
\overline{A_{0}} \hat{z}[1]^{8}+\overline{A_{1}} \hat{z}[1]^{6} \hat{z}[2]+\overline{A_{2}} \hat{z}[1]^{4} \hat{z}[2]^{2}+\overline{A_{1}} \hat{z}[1]^{2} \hat{z}[2]^{3}+\overline{A_{0}} \hat{z}[2]^{4}=0 .
$$

Now take $\sqrt{-1}\left(A_{0}(2.21)-\overline{A_{0}}(2.19)\right)$ and we obtain the following equation with real coefficients

$$
\hat{z}[1]^{2} \hat{z}[2]\left(B_{1} \hat{z}[1]^{4}+B_{2} \hat{z}[1]^{2} \hat{z}[2]+B_{1} \hat{z}[2]^{2}\right)=0
$$

where $B_{1}=2 \Im\left(\overline{A_{0}} A_{1}\right)$ and $B_{2}=2 \Im\left(\overline{A_{0}} A_{2}\right)$ ( $\Im$ stands for the imaginary part of a complex number). Since $\hat{z}[1], \hat{z}[2] \neq 0$, we see that $\hat{z}[2]$ satisfies the real palindromic equation

$$
B_{1} \hat{z}[1]^{4}+B_{2} \hat{z}[1]^{2} \hat{z}[2]+B_{1} \hat{z}[2]^{2}=0
$$

Since the equation is palindromic, if $\hat{z}[2]$ is a root then $1 / \hat{z}[2]=\overline{\hat{z}[2]}$ is also necessarily a root.

At this point we have shown that given $\hat{z}[1]$, there are (at most) two possible values for $\hat{z}[2]$ provided that $B_{1}, B_{2}$ are non-zero. Once we have $\hat{z}[1], \hat{z}[2]$, the values of $\hat{z}[3], \ldots, \hat{z}[L / 2]$ are uniquely determined, assuming that the rational expressions (2.14) and (2.18) are well-defined. However, we have no constraints on $\hat{z}[1]$ other than it lies on the unit circle. Indeed, the polynomial equations (2.12), (2.13), (2.17), (2.23) are weighted homogeneous where the variable $\hat{z}[n]$ has weight $n$. In other words, if $(\hat{z}[1], \hat{z}[2], \hat{z}[3], \ldots, \hat{z}[L / 2])$ is a solution, then $\left(\lambda \hat{z}[1], \lambda^{2} \hat{z}[2], \ldots, \lambda^{L / 2} \hat{z}[L / 2]\right)$ will be a solution for any $\lambda \in S^{1}$. When $L$ is even we obtain a constraint on $\hat{z}[1]$ by noting that $\hat{z}[L / 2]^{2}=1$ since $z[L / 2]=1 / z[L / 2]$ because $L / 2=L-L / 2$. Hence we must have $\left(\lambda^{L / 2}\right)^{2}=1$; i.e., $\lambda^{L}=1$, so $\lambda$ is an $L$-th root of unity. Hence our system can have at most $2 L$ solutions. When $L$ is odd, we observe that $\hat{z}[(L-1) / 2]=\overline{\hat{z}[L-(L-1) / 2]}=\overline{\hat{z}[(L+1) / 2]}=\hat{z}[(L+1) / 2]^{-1}$, and so $\hat{z}[(L-1) / 2] \hat{z}[(L+1) / 2]=1$; and replacing $\hat{z}[k]$ with $\lambda^{k} \hat{z}[k]$, we find $\lambda^{L} \hat{z}[(L-1) / 2] \hat{z}[(L+1) / 2]=1$, i.e., $\lambda^{L}=1$. Hence, our system can only have at most $2 L$ solutions in this case as well.

Generic Conditions. To complete the proof, we explain why for generic $(x, \rho)$ with all $\hat{x}[i]$ non-zero, the quadratic equation (2.23) is non-zero and the rational expressions (2.14) and (2.18) are well-defined. To show that $(2.23)$ is non-vanishing for generic $(x, \rho)$ we must show that $\overline{A_{0}} A_{1}$ and $\overline{A_{0}} A_{2}$ are not pure real. This is a real polynomial condition on $A_{0}, A_{1}, A_{2}$, which are themselves polynomials in the entries of $\rho$ and $x$. To prove that this condition holds generically, it suffices to prove that this is the case for a single choice of $(x, \rho)$. Moreover, since the simplex is Zariski dense in the linear subspace $\sum p[i]+q[i]=1$, it suffices to verify this when the vector $\rho$ lies in this subspace without necessarily being a probability distribution. Applying the Fourier transform, it suffices to verify that the condition holds for a single pair $(\hat{x}, \hat{p})$ with $\hat{p}[0]+\hat{q}[0]=1$. The expressions for $A_{0}, A_{1}, A_{2}$ are determined by the moment entries $M_{1,0}, M_{2,-1}, M_{3,-2}, M_{2,0}, M_{1,1}, M_{3,-2}$, which are in turn determined by the seven values $\hat{x}[1], \hat{x}[2], \hat{x}[3], \hat{p}[1], \hat{p}[2], \hat{q}[L-1], \hat{q}[L-2]$. In particular if we set $\{x[1], x[2], x[3]\}=\{1,1, \sqrt{-1}\},\{\hat{p}[1], \hat{p}[2]\}=\{1,1\}$ and $\hat{\{} q[L-1], q[L-2]\}=\{1+\sqrt{-1}, \sqrt{-1}\}$, then $B_{1}=16$ and $B_{2}=-32$.

Since $\left(z, \rho^{\prime}\right)=(x, \rho)$ automatically satisfies the system of equations $(2.9)$, it follows that $\hat{z}[1]=$ $\lambda x[1]$, where $\lambda$ is an $L$-th root of unity. Moreover, we know that that when $\hat{z}[1]=\hat{x}[1]$, the quadratic equation (2.23) has solutions $\hat{z}[2]=\{\hat{x}[2], 1 / \hat{x}[2]\}$. Hence, if $\hat{z}[1]=\lambda \hat{x}[1]$, then (2.23) has solutions 
$\hat{z}[2]=\left\{\lambda^{2} \hat{x}[2], 1 /\left(\lambda^{2} \hat{x}[2]\right)\right\}$. It follows that the rational expression (2.18) is well-defined as long the polynomial expressions

$$
\left(M_{1,0}^{2} M_{2,0}^{2} \hat{z}[1]^{4}-M_{1,0} M_{1,1}^{2} \hat{z}[1]^{2} \hat{z}[2]-M_{2,0} M_{2,-1} \hat{z}[1]^{2} \hat{z}[2]+M_{1,0} M_{2,0} \hat{z}[2]^{2}\right),
$$

and

$$
\left(M_{1,0} M_{3,-1} \hat{z}[1]^{4}-M_{2,-1} M_{3,-1} \hat{z}[1]^{2} \hat{z}[2]-M_{1,1} M_{3,-2} \hat{z}[1]^{2} \hat{z}[2]+M_{2,0} M_{3,-2} \hat{z}[2]^{2}\right),
$$

are both non-zero when $\{\hat{z}[1], \hat{z}[2]\}=\left\{\lambda \hat{x}[1], \lambda^{2} \hat{x}[2]\right\}$ or $\{\hat{z}[1], \hat{z}[2]\}=\left\{\lambda \hat{x}[1], 1 /\left(\lambda^{2} \hat{x}[2]\right)\right\}$. If this is the case, then it follows that $\hat{z}[3]=\lambda^{3} \hat{x}[3]$ or $\hat{z}[3]=1 /\left(\lambda^{3} \hat{x}[3]\right)$ because we know that $\left(\hat{x}[1], \lambda \hat{x}[2], \lambda^{3} \hat{x}[3]\right)$ and $\left(\hat{x}[1], 1 /\left(\lambda^{2} \hat{x}[2]\right), 1 /\left(\lambda^{2} \hat{x}[3]\right)\right)$ are the first three entries of a vector in the $D_{2 N}$ orbit of the vector $\hat{x}$. Using (2.15) and (2.16), we can now continue recursively to obtain sufficient genericity conditions on the pair $(x, \rho)$.

Remark 2.5. As can be seen from the proof, we only use $\sim 5 L / 2$ of the entries of $M_{i, j}$ (out of $L^{2}$ entries overall) to determine the orbit of $x$. Precisely, we only use the $\sim 5 L / 2$ entries $M_{\ell,-\ell}, M_{\ell, 1-\ell}, M_{\ell+1,0}, M_{\ell, 1}$ and $M_{\ell-1,2}$ for $\ell=0, \ldots, L / 2$. A similar observation was made in [16].

\subsection{General theory for MRA with a general distribution over finite groups}

The purpose of this section is to discuss the theory of moments for the MRA problem for finite groups. Our goal is to highlight the mathematical differences between uniform and generic distributions on the group $G$. Precisely, the dihedral MRA model (1.1) we consider here is a special case of the following MRA problem:

Recover a signal $x \in V$ from moment measurements of $g_{i} \cdot x+\epsilon_{i}$, where the group elements $g_{i}$ are chosen 'at random' from a finite group $G$ and $V$ is a finite dimensional vector space.

\subsubsection{Uniform distribution}

The case of a uniform distribution of the group elements $g \in G$ was studied in depth in [8]. For the uniform distribution, the $n$-th moment

$$
M^{n}=\frac{1}{|G|} \sum_{g \in G} g x^{\otimes n},
$$

is a tensor whose components generate the vector space of invariant polynomial functions of degree $n$ on $V$. An important theoretical result whose proof uses Jennrich's algorithm for decomposing a three-tensor is the following theorem:

Theorem. [8, Theorem D.2] Let $G$ be a finite group and let $V$ be the regular representation of $G$ over $\mathbb{R}$, then the generic orbit $G x$ consists of linearly independent vectors and consequently generic recovery is possible from degree 3 invariants.

(The regular representation of a finite group is the $|G|$ dimensional vector space of functions $G \rightarrow \mathbb{R}$ where the group $G$ acts by $(g \circ f)(h)=f\left(g^{-1} h\right)$.) 
Since $\mathbb{R}^{L}$ is the regular representation of the cyclic group $\mathbb{Z}_{L}$, the Theorem above implies that for the uniform distribution on $\mathbb{Z}_{L}$ the generic vector $x \in \mathbb{R}^{L}$ can be recovered from the third order moment: a result originally proved in $[14,39]$. Note, however, that this result cannot be applied for the action of $D_{2 L}$ on $\mathbb{R}^{L}$ because $\mathbb{R}^{L}$ is not the regular representation of $D_{2 L}$ since its dimension is smaller than the order of the group $D_{2 L}$. As a result, we do not know if the first three moments suffice to recover a generic orbit when the distribution in $D_{2 L}$ is uniform.

\subsubsection{Generic distributions}

We now give a theoretical analysis of the situation where the group elements $g_{i}$ are taken from a generic distribution on the finite group $G$, as we do here for the dihedral group $D_{2 L}$ and as was done in [2] for the cyclic group $\mathbb{Z}_{L}$.

Observe that a probability distribution on a finite group is a function $\rho: G \rightarrow \mathbb{R}$ satisfying the conditions $\rho(g) \geq 0$ for all $g \in G$ and $\sum_{g \in G} \rho(g)=1$. Thus, a probability distribution is a vector $\rho$ in the regular representation which lies in the simplex $\Delta_{|G|} \subset R(G)$, where $R(G)$ denotes the regular representation. By definition, the $n$-th order moment associated to a probability distribution $\rho$ on $G, M^{n}:=\sum_{g \in G} \rho(g)(g x)^{\otimes n}$ is a $n$-tensor of invariant polynomials of bidegree $(1, n)$ on $R(G) \times V$. Of particular interest in this paper is the second order moment $M^{2}(x, \rho)=\sum_{g \in G} \rho(g)(g x)(g x)^{T}$, when $G$ is the dihedral group. In this case, the second order moment gives a collection of invariant functions of total degree 3 on $R(G) \times V$.

The following result which is of purely theoretical interest states that the orbit of a generic pair $(\rho, x) \in R(G)$ can be determined from the full collection of degree 3 invariant polynomials.

Proposition 2.6. The set of all degree 3 invariants on $R(G) \times V$ determines the $G$-orbit of a generic pair $(\rho, x)$.

Proof. As in [8] it suffices to show that the orbit of a generic $(\rho, x) \in R(G) \times V$ consists of linearly independent vectors. Note that projection map $R(G) \times V \rightarrow R(G)$ is $G$-invariant. Thus the projection of the orbit $G(\rho, x)$ to $R(G)$ is the $G$-orbit of $\rho$ in $R(G)$. It then follows from [8, Theorem D.2] that $G \rho$ consists of linearly independent vectors and hence so does $G(\rho, x)$. We can then recover the orbit from degree three invariants.

Remark 2.7. Note that there is no way to estimate all of the degree invariants in $R(G) \times V$ from a given set of MRA measurements. For this reason, Proposition 2.6 is only of theoretical interests. In particular, note that even from a theoretical point of view our results for the dihedral group acting on $V=\mathbb{R}^{L}$ are much stronger that the guarantee given by Proposition 2.6 since they state that quite a small subset of the degree three invariants of $R(G) \times V$ are sufficient to recover generic orbits.

List recovery. Following the terminology of [8, Section 1.4], we say that a signal $x$ admits list recovery from a set of moment measurements if there are a finite number of orbits with same moments. As was done in [8, Section 4.2.2], one can use the Jacobian criterion to determine if a collection of MRA moments with generic distribution allows list recovery for a generic orbit $x$.

Precisely, let $f_{1}, \ldots, f_{r} \in \mathbb{R}\left[p_{1}, \ldots, p_{g}, x_{1}, \ldots, x_{L}\right]^{G}$ be a collection of invariant polynomials of degrees $\left(1, d_{1}\right), \ldots,\left(1, d_{r}\right)$ corresponding to some set of entries of the moment tensors $M^{d_{1}}(\rho, x), \ldots, M^{d_{r}}(\rho, x)$. Then these moments are sufficient to allow list recovery of a generic 
signal if and only if the rank of the Jacobian matrix $J\left(f_{1}, \ldots, f_{r}\right)$ equals $|G|-1+L$. The rank of the Jacobian can be effectively computed in examples, but this will be considered in another work.

An easy consequence of the Jacobian criterion is the following corollary.

Corollary 2.8. If $|G|>\left(\begin{array}{c}L+1 \\ 2\end{array}\right)+L$, then list recovery is impossible from second order moments.

Proof. Since the second order moment tensor is symmetric, the total number of first and second order moments is $L+\left(\begin{array}{c}L+1 \\ 2\end{array}\right)$ which is smaller than $G-1+L$ so list recovery is impossible.

Orbit recovery. Using methods from algebraic geometry we can also give a criterion for when a collection of moment polynomials allows for generic orbit recovery. However, this criterion involves computing the dimension and degree of an algebraic variety. Such calculations can be done symbolically using a computer algebra system but not efficiently [15, Appendix D].

To simplify the discussion we focus on the first and second order moments and recall the strategy used in the proof of Theorem 2.3. Given a generic probability distribution $\rho=\left\{p_{g}\right\}_{g \in G=D_{2 L}}$ and a generic vector $x \in V=\mathbb{R}^{L}$, we proved that the following system of bilinear equations in the $3 L-1$ unknowns $x^{\prime}, p_{g}^{\prime}$ has at most $2 L=|G|$ solutions

$$
\begin{array}{cl}
\sum_{g} p_{g} g x-p_{g}^{\prime} g x^{\prime} & =0 \\
\sum_{g}\left(p_{g}(g x)^{T} g x-p_{g}^{\prime}\left(g x^{\prime}\right)^{T} g x^{\prime}\right) & =0 .
\end{array}
$$

(Note that the number of unknowns is $3 L-1$ because $\sum_{g \in G} p_{g}^{\prime}=1$ since $\rho^{\prime}$ is a probability distribution and we can therefore express one of the $p_{g}$ in terms of the other ones.) The next proposition shows that our verification was equivalent to proving a statement about an incidence variety associated to the group $G$ and vector space $V=\mathbb{R}^{L}$. To formulate the result, we first establish notation for the action of a finite group $G$ on a vector space $V$. Let $I \subset(R(G) \times V)^{2}$ be the subvariety defined by the bilinear equations (2.24), where the $x, x^{\prime},\left\{p_{g}\right\},\left\{p_{g}^{\prime}\right\}$ are all considered variables. Since $\rho, \rho^{\prime}$ are probability distributions $\sum_{g \in G} p_{g}=\sum_{g \in G} p_{g}^{\prime}$ so we can view this as a system of equations in $2(\operatorname{dim} V+|G|-1)$ variables.

In the language of algebraic geometry, $I$ is called an incidence variety. The geometry of the incidence variety $I$ characterizes when orbit and list recovery are possible.

Proposition 2.9. Let $G$ be a finite group acting on a vector space $V$, and let $I \subset(R(G) \times V)^{2}$ be an incidence defined in (2.24).

1. If $\operatorname{dim} I=\operatorname{dim} V+|G|-1$, then for a generic signal $x$ and probability distribution $\rho=\left\{p_{g}\right\}_{g \in G}$, list recovery is possible from the first and second order moments $M^{2}(x, \rho)$.

2. If $\operatorname{dim} I=\operatorname{dim} V+|G|$ and in addition $\operatorname{deg} I=|G|$, then for a generic signal $x$ and probability distribution $\rho=\left\{p_{g}\right\}_{g \in G}$, orbit recovery is possible from the first and second orders moment $M^{2}(x, \rho)$.

Proof. Consider the projection $\pi: I \rightarrow R(G) \times V$ defined by $\left(x, \rho, x^{\prime}, \rho^{\prime}\right) \mapsto(x, \rho)$. If $\operatorname{dim} I=$ $\operatorname{dim} V+R(G)-1$, then the generic fiber of $\pi$ must be 0 -dimensional. Hence, for a generic vector $x \in V$ and probability distribution $\rho \in R(G)$, there can be at most a finite number of pairs $\left(x, \rho, x^{\prime}, \rho^{\prime}\right) \in I$. In other words, there are finite number of vectors $x^{\prime}$ such that there exist a distribution $\rho^{\prime}$ with the property that $M^{1}(x, \rho)=M^{1}\left(x^{\prime}, \rho^{\prime}\right)$ and $M^{2}(x, \rho)=M^{2}\left(x^{\prime}, \rho^{\prime}\right)$ This proves part (i). 
Note that for each $g \in G$, the set $X_{g}=\{(x, \rho, g x, g \rho) \mid x \in V, \rho \in R(G)\}$ is a $\operatorname{dim} V+|G|-1$ dimensional subvariety of $I$, which is isomorphic to $R(G) \times V$. In particular, if $\operatorname{dim} I=\operatorname{dim} V+|G|$ then it must necessarily be an irreducible component of the variety $V$ in the sense of algebraic geometry. Hence, if $\operatorname{dim} I=\operatorname{dim} V+|G|-1$ then $I$ has at least $|G|$ irreducible components. and therefore its degree must be at least $|G|$. Hence, if $\operatorname{dim} I=\operatorname{dim}|V|+|G|-1$ and $\operatorname{deg} I=|G|$, then $I$ has exactly $|G|$ irreducible components and for generic $x, \rho$ there will be exactly $|G|$ pairs $\left(x, \rho, x^{\prime}, \rho^{\prime}\right) \in I$. Hence each $x^{\prime}$ must necessarily equal $g x$ for some $g \in G$. Therefore, the first and second order moments recover generic orbits $x$ in this case.

\section{Algorithms}

In this section, we introduce three algorithmic paradigms to estimate the signal $x$ from dihedral MRA observations $y_{1}, \ldots, y_{n}$ as in (1.2). We first introduce the three methods, and then compare them numerically in Section 3.4.

\subsection{Group synchronization}

If the group elements $g_{1}, \ldots, g_{n} \in D_{2 L}$ were known, estimating the signal can be done by aligning the observations and averaging out the noise:

$$
x_{\mathrm{est}}=\frac{1}{n} \sum_{i=1}^{n} g_{i}^{-1} y_{i} .
$$

This motivates synchronization methods to estimate the unknown group elements from the observations. Synchronization starts by aligning all pairs of observations $y_{i}, y_{j}, i \neq j$, so that

$$
y_{i} \approx g_{i j} y_{j}
$$

for some group element $g_{i j} \in D_{2 L}$. A standard alignment procedure is based on cross-correlating the observations. In more general groups, other common features can be harnessed; see for example [31, 50]. The relation (3.2) is merely a proxy to $g_{i} \cdot x \approx g_{i j} g_{j} \cdot x$, which in turn means that $g_{i} g_{j}^{-1} \approx g_{i j}$. At this stage, one reduces the MRA problem to the problem of group synchronization [49], where we aim at estimating the unknown group elements $g_{1}, \ldots, g_{n}$ from a subset of their ratios $g_{i} g_{j}^{-1}$, often corrupted with noise.

Early synchronization studies addressed the problem over compact groups, such as, finite groups, phases, and rotations. The common property of all synchronization cases over compact groups is that we can reduce them all to synchronization over rotations, or a subgroup of rotations, by using a faithful orthogonal representation [20, 23, 40, 52]. Further generalizations extended synchronization methods to non-compact groups, and in particular to the Euclidean group, see e.g., $[38,44,17]$.

Specifically for the dihedral MRA problem (1.1), we start by computing the cross-correlation between any observation $y_{i}$ and any other observation $y_{j}$ and its reflection $s y_{j}$. The maximal value indicates the best alignment as in (3.2). The resulting ratios $g_{i j} \approx g_{i} g_{j}^{-1}$ serve as an input for a standard spectral algorithm [49], which uses a rounding procedure onto the dihedral group, 
resulting in estimates of the group elements $\tilde{g}_{1}, \ldots, \tilde{g}_{n}$. The orbit of the signal is then estimated by averaging over the synchronized observations

$$
x_{\mathrm{est}}=\frac{1}{n} \sum_{i=1}^{n} \tilde{g}_{i}^{-1} y_{i}
$$

Unfortunately, in low SNR environments the error of estimating the ratios $g_{i} g_{j}^{-1}$, and thus of estimating $g_{1}, \ldots, g_{n}$, grows rapidly $[5,42,41,13]$. Thus, in such regimes we consider techniques which aim to recover the signal $x$ directly, bypassing the estimation of the missing group elements $\left\{g_{i}\right\}_{i=1}^{n}$. Next, we present two such methods, based on expectation-maximization and the method of moments.

\subsection{Maximum likelihood estimation using expectation-maximization}

The log-likelihood function of (1.1) is given by

$$
\ell(x, \rho)=\log p\left(y_{1}, \ldots, y_{n} ; x, \rho\right)=\sum_{i=1}^{N} \log \sum_{j=1}^{2 L} \rho[j] \frac{1}{\left(2 \pi \sigma^{2}\right)^{L / 2}} e^{-\frac{\left\|y_{i}-g[j] \cdot x\right\|^{2}}{2 \sigma^{2}}}
$$

where $g[1], \ldots, g[2 L]$ are the elements of $D_{2 L}$. This is the standard likelihood function of a Gaussian mixture model, but all centers are connected through the orbit of $D_{2 L}$ acting on $x$. We wish to find the signal $x$ and distribution $\rho$ that maximize (3.4). In the sequel, we assume no prior information on the signal and the distribution. If such information is available, then it is useful to consider the $\log$-posterior distribution $\log p\left(x, \rho \mid y_{1}, \ldots, y_{n}\right)$, which is equal to the log-likelihood plus the log of the prior terms.

To maximize the likelihood function, we devise an expectation-maximization (EM) algorithm [25]. The EM algorithm has been successfully applied to other MRA setups [14, 2, 36, 33] as well as for cryo-EM $[45,48,12]$. Although EM is not guaranteed to achieve the maximum of the non-convex likelihood function (3.4), it is guaranteed that each EM iteration does not reduce the likelihood. In addition, for the general discrete MRA model, it was shown that at low noise, this landscape is "benign", namely, there are no spurious local optima (besides the maximum likelihood) and only strict saddle points. At high noise, this landscape may develop spurious local optima, depending on the specific group. In addition, it was shown that the likelihood landscape is locally convex [27].

EM is an iterative algorithm, and each step consists of two steps. In the first step, called the E-step, the expectation of the complete likelihood (namely, the joint likelihood of $x, \rho$ and the group elements) is computed. The expectation is taken with respect to the group elements (i.e., the nuisance variables), given the current estimates of the signal $x_{t}$ and the distribution $\rho_{t}$ :

$$
\begin{aligned}
Q\left(x, \rho \mid x_{t}, \rho_{t}\right) & =\mathbb{E}\left\{\log p\left(y_{1}, \ldots, y_{n}, g_{1}, \ldots, g_{n} ; x, \rho\right)\right\} \\
& =\sum_{i=1}^{n} \mathbb{E}\left\{-\frac{1}{2 \sigma^{2}}\left\|y_{i}-g_{i} \cdot x\right\|^{2}+\log \rho\left[g_{i}\right]\right\}+\text { constant } \\
& =\sum_{i=1}^{N} \sum_{j=1}^{2 L} w_{i, j}\left\{-\frac{1}{2 \sigma^{2}}\left\|y_{i}-g[j] \cdot x\right\|^{2}+\log \rho[j]\right\}+\text { constant }
\end{aligned}
$$


where

$$
w_{i, j}=\frac{\rho_{t}[j] e^{\frac{-1}{2 \sigma^{2}}\left\|y_{i}-g[j] \cdot x_{t}\right\|^{2}}}{\sum_{j=1}^{2 L} \rho_{t}[j] e^{\frac{-1}{2 \sigma^{2}}\left\|y_{i}-g[j] \cdot x_{t}\right\|^{2}}} .
$$

The second step, called M-step, maximizes $Q$ with respect to $x$ and $\rho$. In our case, the update step reads:

$$
\begin{gathered}
x_{t+1}=\frac{1}{n} \sum_{i=1}^{N} \sum_{j=1}^{2 L} w_{i, j} g^{-1}[j] y_{i} \\
\rho_{t+1}[j]=\frac{\sum_{i=1}^{n} w_{i, j}}{\sum_{i=1}^{n} \sum_{j=1}^{2 L} w_{i, j}} .
\end{gathered}
$$

If prior information is available (and thus the EM tries to maximize the posterior distribution rather than the likelihood), then it will act as a regularizer on the solution of the M-step. The EM algorithm iterates between computing the weights (3.6) and updating the parameters (3.7) until a stopping criterion is met.

\subsection{The method of moments}

The idea behind the method of moments is finding a pair $(x, \rho)$ whose moments match the empirical moments of the observations. In particular, according to Theorem 1.1, only the first two moments are required to uniquely characterize the orbit of generic $x$ and $\rho$. The empirical moments can be computed from the data simply by averaging:

$$
\begin{aligned}
& M_{\mathrm{est}}^{1}=\frac{1}{n} \sum_{i=1}^{n} y_{i}, \\
& M_{\mathrm{est}}^{2}=\frac{1}{n} \sum_{i=1}^{n} y_{i} y_{i}^{T} .
\end{aligned}
$$

By the law of large numbers, and using Lemma 2.2, for large $n$ we have

$$
\begin{array}{r}
M_{\mathrm{est}}^{1} \approx M^{1}(x, \rho)=C_{p} x+C_{q} s x, \\
M_{\mathrm{est}}^{2} \approx M^{2}(x, \rho)=C_{x} D_{p} C_{x}^{T}+C_{s x} D_{q} C_{s x}^{T},
\end{array}
$$

where $C_{z} \in \mathbb{R}^{L \times L}$ is a circulant matrix generated by $z \in \mathbb{R}^{L}$, and $D_{z} \in \mathbb{R}^{L \times L}$ is a diagonal matrix whose entries are $z$. As $n \rightarrow \infty, M_{\text {est }}^{1} \rightarrow M^{1}(x, \rho)$ and $M_{\text {est }}^{2} \rightarrow M^{2}(x, \rho)$ almost surely.

A common practice is to estimate $(x, \rho)$ from $M_{\text {est }}^{1}$ and $M_{\text {est }}^{2}$ by minimizing a non-convex least squares objective:

$$
\min _{\tilde{x} \in \mathbb{R}^{L},[p, q] \in \Delta^{2 L}}\left\|M_{\mathrm{est}}^{2}-C_{\tilde{x}} D_{p} C_{\tilde{x}}^{T}-C_{s \tilde{x}} D_{q} C_{s \tilde{x}}^{T}\right\|_{\mathrm{F}}^{2}+\lambda\left\|M_{\mathrm{est}}^{1}-C_{p} \tilde{x}-C_{q} s \tilde{x}\right\|_{2}^{2} .
$$

The solution of (3.10) is the method of moments estimator. While the objective function (3.10) is non-convex, it seems to provide accurate estimates in many cases. In the low SNR regime, the method of moments is tightly connected to the maximum likelihood estimator. Specifically, in this regime likelihood optimization reduces to a sequence of least squares optimization problems that match moments [35, 26]. Since we use only two moments, the method of moments (3.10) can be interpreted as an approximation of the maximum likelihood estimator. 


\subsection{Numerical experiments}

This section compares numerically the algorithmic methods discussed above: synchronization, expectation-maximization, and the method of moments. We define signal-to-noise ratio (SNR) as $\|x\|^{2} /\left(L \sigma^{2}\right)$. To account for the group symmetry, we define relative error as

$$
\text { relative error }=\min _{g \in D_{2 L}} \frac{\left\|g \cdot x_{\mathrm{est}}-x\right\|}{\|x\|},
$$

where $x_{\text {est }}$ is the signal estimate. The entries of the ground-truth $x$ of length $L=10$ were drawn i.i.d. from a normal distribution with mean zero and variance one, and the distribution $\rho$ was uniformly sampled from the simplex $\Delta_{2 L}$.

We consider two regimes: (i) a relatively small number of observations $(n=1000)$ and moderate SNR levels, and (ii) large $n$ and low SNR. The code to reproduce all experiments is publicly available at https://github.com/nirsharon/DihedralMRA. The results below represent the average over 50 trials. We initialized the EM algorithm from a single random point and halted it when the difference of the likelihood between two consecutive iterations dropped below $10^{-4}$, or after a maximum of 400 iterations. For the method of moments, we minimized (3.10) using the trust-regions method; we initialized the optimization algorithm from 10 different random initial guesses and chose the one that yields the least value of the cost (3.10). The number of trust-regions iterations was limited to 200.

Moderate SNR regime. We begin with a noise regime where the synchronization approach presents a viable alternative to EM and the method of moments. Figure 2 shows the relative error of the three methods as a function of the SNR with $n=1000$ observations. The method of moments shows inferior results compared to synchronization and EM since the empirical moments do not approximate the population moments accurately enough for such a small number of observations. For high SNR, the performance of synchronization and EM are comparable. The synchronization behavior is thus compatible with current knowledge about the synchronization problem and the spectral algorithm specifically, see, e.g., [24, 29]. However, as the SNR drops, synchronization fails to estimate the group elements accurately, while both the method of moments and EM present consistent error rates. This phenomenon agrees with theoretical findings regarding alignment in the presence of high noise [5] and synchronization when applied to such corrupted input data [50]. As the SNR approaches 1, when the signal and the noise are of the same order, the synchronization method introduces relative error close to 1, meaning it contributes no information about the solution.

Low SNR. We discard the synchronization algorithm in the low SNR regime as it cannot cope with high noise levels, as demonstrated in Figure 2. In addition, since the first step of the synchronization method involves pairwise alignment, the synchronization input consists of $\mathcal{O}\left(n^{2}\right)$ group elements, and so the computational complexity of this method makes it impractical for as many as $n=10^{5}$ observations.

Figure 3a shows relative errors as a function of SNR. The EM outperforms the method of moments for SNR values above 1/10. For lower SNR levels, the method of moments shows similar estimation rates. In the high SNR regime, the error curves of both methods scale as $\mathrm{SNR}^{-1 / 2}$, namely as $\sigma$, which is the same estimation rate as if the group elements were known. In particular, 


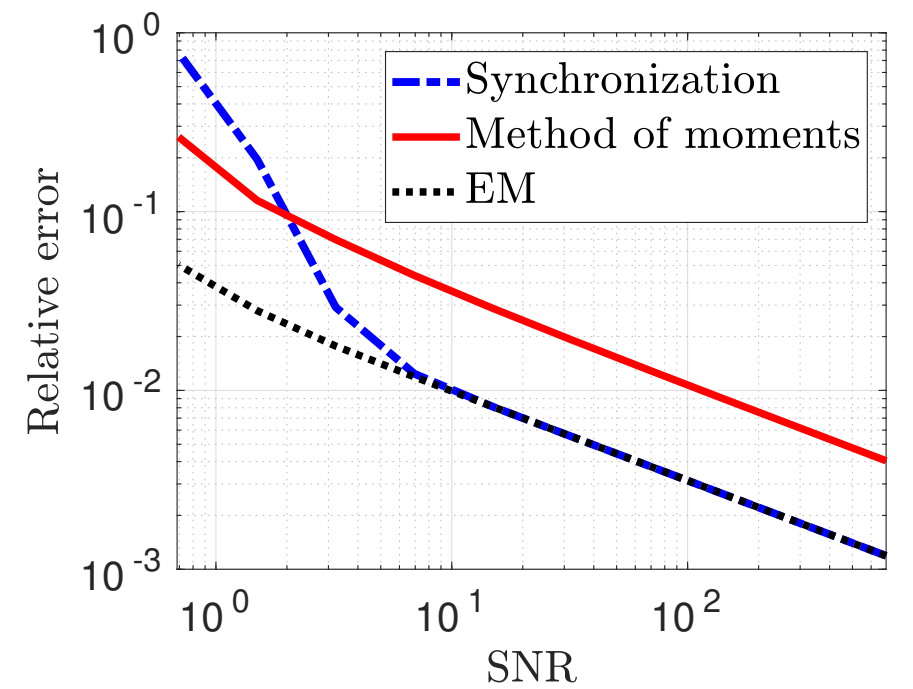

Figure 2: The relative error of the three methods under moderate SNR levels with $n=1000$ observations. As the SNR deteriorates, the synchronization method fails to estimate the group elements, and thus the signal, accurately.

the numerical slope of the EM method is -0.4999 and the method of moments presents a numerical slope of -0.5104 . In the low SNR regime, however, the error curves scale as $\mathrm{SNR}^{-1} \propto \sigma^{2}$. While this slope is expected for the method of moments that directly uses the first two moments (and thus its standard deviation is proportional to $\sigma^{2}$ ), the moments do not appear explicitly in the EM iterations. Specifically, the numerical slopes for SNR values below 1/10 were -1.0561 and -1.1058 for the method of moments and EM, respectively. This rate implies that accurate estimation requires $n \gg \sigma^{4}$, corroborating our theoretical findings (Corollary 1.2) that no algorithm can achieve better estimation rates in the low SNR regime. A similar phenomenon was observed by previous MRA studies [48, 14, 2, 18]. For the connection between EM and the method of moments in the low SNR regime, see [35, 27, 26].

Figure $3 \mathrm{~b}$ presents the corresponding average runtime. The runtime of EM increases as the SNR decreases, while the runtime of the method of moments remains roughly constant. The reason for the growth in runtime is revealed in Figure 4, where we display the average number of EM iterations as a function of SNR. The figure shows that the number of iterations is inversely proportional to the SNR.

\section{Acknowledgment}

W.L. and N.S. are partially supported by BSF grant no. 2018230. T.B. and D.E. are partially supported by BSF grant no. 2020159. T.B. and N.S are partially supported by the NSF-BSF award 2019752. T.B. is also supported in part by the ISF grant no. 1924/21. D.E. is supported by Simons Collaboration grant 708560. W.L. is partially supported by NSF award IIS- 1837992 . 


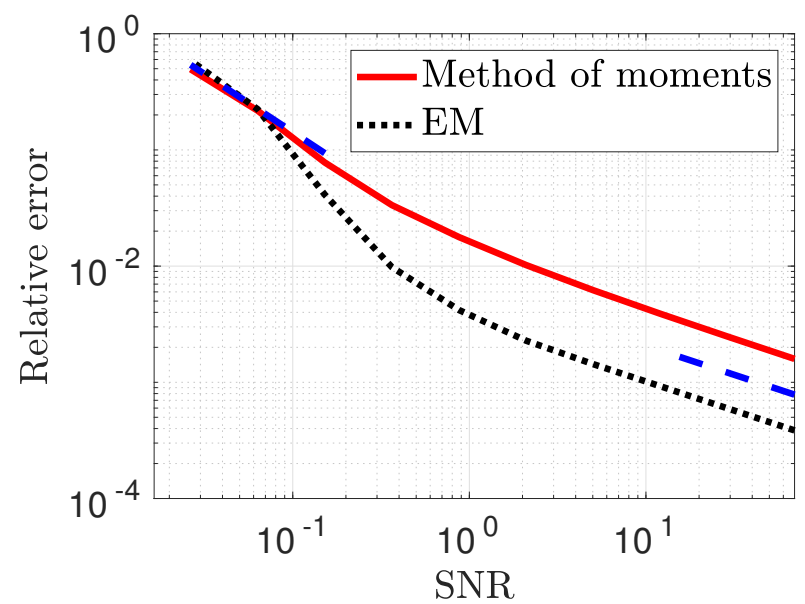

(a)

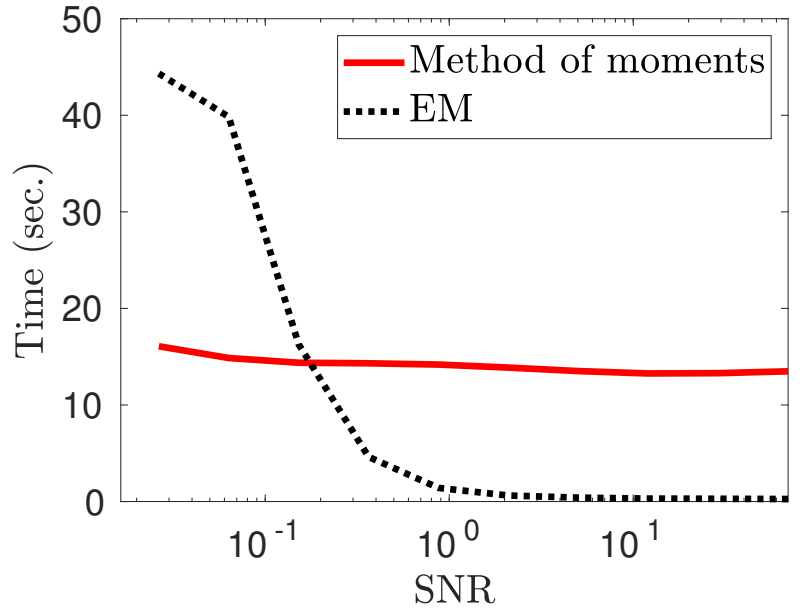

(b)

Figure 3: Relative error (left panel) and runtime (right panel) of the method of moments and EM as a function of the SNR for $n=10^{5}$ observations. In the high SNR regime, the slope of the error curves (dashed blue line), for both methods, scales as $\mathrm{SNR}^{-1 / 2} \propto \sigma$, which is the same estimation rate as if the group elements were known. In the low SNR regime, however, the error curves scale as $\mathrm{SNR}^{-1} \propto \sigma^{2}$, corroborating our theoretical findings.

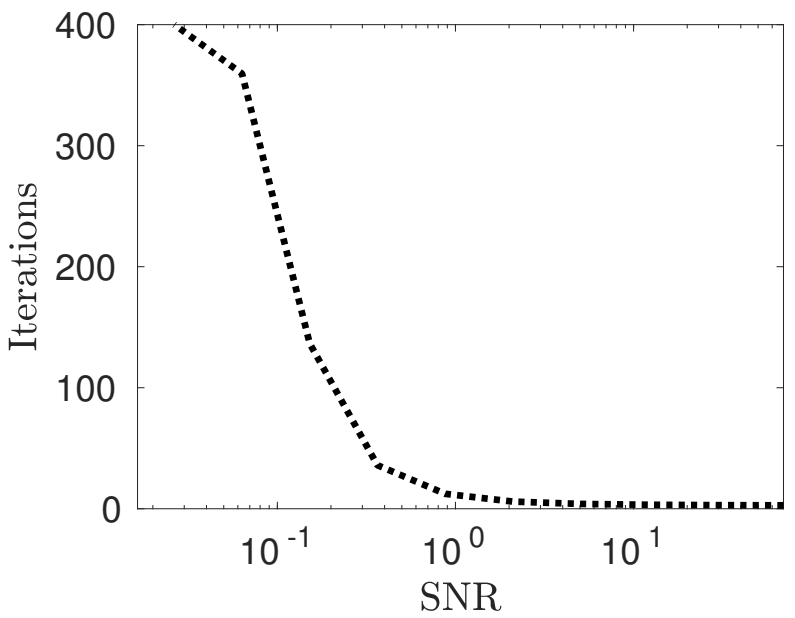

Figure 4: The average number of EM iterations as a function of the SNR. The maximum number of iterations is set to 400 . 


\section{References}

[1] Asaf Abas, Tamir Bendory, and Nir Sharon. The generalized method of moments for multireference alignment. arXiv preprint arXiv:2103.02215, 2021.

[2] Emmanuel Abbe, Tamir Bendory, William Leeb, João M Pereira, Nir Sharon, and Amit Singer. Multireference alignment is easier with an aperiodic translation distribution. IEEE Transactions on Information Theory, 65(6):3565-3584, 2018.

[3] Emmanuel Abbe, João M Pereira, and Amit Singer. Sample complexity of the boolean multireference alignment problem. In 2017 IEEE International Symposium on Information Theory (ISIT), pages 1316-1320. IEEE, 2017.

[4] Emmanuel Abbe, João M Pereira, and Amit Singer. Estimation in the group action channel. In 2018 IEEE International Symposium on Information Theory (ISIT), pages 561-565. IEEE, 2018.

[5] Cecilia Aguerrebere, Mauricio Delbracio, Alberto Bartesaghi, and Guillermo Sapiro. Fundamental limits in multi-image alignment. IEEE Transactions on Signal Processing, 64(21):5707$5722,2016$.

[6] Yariv Aizenbud, Boris Landa, and Yoel Shkolnisky. Rank-one multi-reference factor analysis. Statistics and Computing, 31(1):1-31, 2021.

[7] Philip R Baldwin and Dmitry Lyumkis. Non-uniformity of projection distributions attenuates resolution in cryo-EM. Progress in biophysics and molecular biology, 150:160-183, 2020.

[8] Afonso S Bandeira, Ben Blum-Smith, Joe Kileel, Amelia Perry, Jonathan Weed, and Alexander S Wein. Estimation under group actions: recovering orbits from invariants. arXiv preprint arXiv:1712.10163, 2017.

[9] Afonso S Bandeira, Moses Charikar, Amit Singer, and Andy Zhu. Multireference alignment using semidefinite programming. In Proceedings of the 5th conference on Innovations in theoretical computer science, pages 459-470, 2014.

[10] Afonso S Bandeira, Yutong Chen, Roy R Lederman, and Amit Singer. Non-unique games over compact groups and orientation estimation in cryo-EM. Inverse Problems, 36(6):064002, 2020 .

[11] Afonso S Bandeira, Jonathan Niles-Weed, and Philippe Rigollet. Optimal rates of estimation for multi-reference alignment. Mathematical Statistics and Learning, 2(1):25-75, 2020.

[12] Tamir Bendory, Alberto Bartesaghi, and Amit Singer. Single-particle cryo-electron microscopy: Mathematical theory, computational challenges, and opportunities. IEEE Signal Processing Magazine, 37(2):58-76, 2020.

[13] Tamir Bendory, Nicolas Boumal, William Leeb, Eitan Levin, and Amit Singer. Multi-target detection with application to cryo-electron microscopy. Inverse Problems, 35(10):104003, 2019. 
[14] Tamir Bendory, Nicolas Boumal, Chao Ma, Zhizhen Zhao, and Amit Singer. Bispectrum inversion with application to multireference alignment. IEEE Transactions on Signal Processing, 66(4):1037-1050, 2017.

[15] Tamir Bendory and Dan Edidin. Toward a mathematical theory of the crystallographic phase retrieval problem. SIAM Journal on Mathematics of Data Science, 2(3):809-839, 2020.

[16] Tamir Bendory, Dan Edidin, and Shay Kreymer. Signal recovery from a few linear measurements of its high-order spectra. Applied and Computational Harmonic Analysis, 56:391-401, 2022 .

[17] Tamir Bendory, Ido Hadi, and Nir Sharon. Compactification of the rigid motions group in image processing. arXiv preprint arXiv:2106.13505, 2021.

[18] Tamir Bendory, Ariel Jaffe, William Leeb, Nir Sharon, and Amit Singer. Super-resolution multi-reference alignment. Information and Inference: A Journal of the IMA.

[19] Tamir Bendory, Oscar Mickelin, and Amit Singer. Sparse multi-reference alignment: sample complexity and computational hardness. arXiv preprint arXiv:2109.11656, 2021.

[20] Nicolas Boumal. Nonconvex phase synchronization. SIAM Journal on Optimization, 26(4):2355-2377, 2016.

[21] Nicolas Boumal, Tamir Bendory, Roy R Lederman, and Amit Singer. Heterogeneous multireference alignment: A single pass approach. In 2018 52nd Annual Conference on Information Sciences and Systems (CISS), pages 1-6. IEEE, 2018.

[22] Victor-Emmanuel Brunel. Learning rates for Gaussian mixtures under group action. In Conference on Learning Theory, pages 471-491. PMLR, 2019.

[23] Luca Carlone, Roberto Tron, Kostas Daniilidis, and Frank Dellaert. Initialization techniques for 3D SLAM: a survey on rotation estimation and its use in pose graph optimization. In 2015 IEEE international conference on robotics and automation (ICRA), pages 4597-4604. IEEE, 2015.

[24] Mihai Cucuringu. Sync-rank: Robust ranking, constrained ranking and rank aggregation via eigenvector and SDP synchronization. IEEE Transactions on Network Science and Engineering, 3(1):58-79, 2016.

[25] Arthur P Dempster, Nan M Laird, and Donald B Rubin. Maximum likelihood from incomplete data via the EM algorithm. Journal of the Royal Statistical Society: Series B (Methodological), 39(1):1-22, 1977.

[26] Zhou Fan, Roy R Lederman, Yi Sun, Tianhao Wang, and Sheng Xu. Maximum likelihood for high-noise group orbit estimation and single-particle cryo-EM. arXiv preprint arXiv:210\%.01305, 2021.

[27] Zhou Fan, Yi Sun, Tianhao Wang, and Yihong Wu. Likelihood landscape and maximum likelihood estimation for the discrete orbit recovery model. arXiv preprint arXiv:2004.00041, 2020 . 
[28] Chao Gao and Anderson Y Zhang. Iterative algorithm for discrete structure recovery. arXiv preprint arXiv:1911.01018, 2019.

[29] Tingran Gao, Jacek Brodzki, and Sayan Mukherjee. The geometry of synchronization problems and learning group actions. Discrete $8 \mathcal{6}$ Computational Geometry, pages 1-62, 2019.

[30] Subhro Ghosh and Philippe Rigollet. Multi-reference alignment for sparse signals, uniform uncertainty principles and the beltway problem. arXiv preprint arXiv:2106.12996, 2021.

[31] Klaus Häming and Gabriele Peters. The structure-from-motion reconstruction pipeline-a survey with focus on short image sequences. Kybernetika, 46(5):926-937, 2010.

[32] Matthew Hirn and Anna Little. Wavelet invariants for statistically robust multi-reference alignment. Information and Inference: A Journal of the IMA.

[33] Noam Janco and Tamir Bendory. An accelerated expectation-maximization for multi-reference alignment. arXiv preprint arXiv:2105.07372, 2021.

[34] Ramakrishna Kakarala. Completeness of bispectrum on compact groups. arXiv preprint arXiv:0902.0196, 1, 2009.

[35] Anya Katsevich and Afonso Bandeira. Likelihood maximization and moment matching in low SNR gaussian mixture models. arXiv preprint arXiv:2006.15202, 2020.

[36] Chao Ma, Tamir Bendory, Nicolas Boumal, Fred Sigworth, and Amit Singer. Heterogeneous multireference alignment for images with application to 2D classification in single particle reconstruction. IEEE Transactions on Image Processing, 29:1699-1710, 2019.

[37] Katerina Naydenova and Christopher J Russo. Measuring the effects of particle orientation to improve the efficiency of electron cryomicroscopy. Nature Communications, 8(1):1-5, 2017.

[38] Onur Ozyesil, Nir Sharon, and Amit Singer. Synchronization over Cartan motion groups via contraction. SIAM Journal on Applied Algebra and Geometry, 2(2):207-241, 2018.

[39] Amelia Perry, Jonathan Weed, Afonso S Bandeira, Philippe Rigollet, and Amit Singer. The sample complexity of multireference alignment. SIAM Journal on Mathematics of Data Science, 1(3):497-517, 2019.

[40] Amelia Perry, Alexander S Wein, Afonso S Bandeira, and Ankur Moitra. Message-passing algorithms for synchronization problems over compact groups. Communications on Pure and Applied Mathematics, 71(11):2275-2322, 2018.

[41] Dirk Robinson, Sina Farsiu, and Peyman Milanfar. Optimal registration of aliased images using variable projection with applications to super-resolution. The Computer Journal, 52(1):3142, 2009.

[42] Dirk Robinson and Peyman Milanfar. Fundamental performance limits in image registration. IEEE Transactions on Image Processing, 13(9):1185-1199, 2004. 
[43] Elad Romanov, Tamir Bendory, and Or Ordentlich. Multi-reference alignment in high dimensions: sample complexity and phase transition. SIAM Journal on Mathematics of Data Science, 3(2):494-523, 2021.

[44] David M Rosen, Luca Carlone, Afonso S Bandeira, and John J Leonard. SE-Sync: A certifiably correct algorithm for synchronization over the special Euclidean group. The International Journal of Robotics Research, 38(2-3):95-125, 2019.

[45] Sjors HW Scheres. RELION: implementation of a Bayesian approach to cryo-EM structure determination. Journal of structural biology, 180(3):519-530, 2012.

[46] Jean-Pierre Serre. Linear representations of finite groups. Graduate Texts in Mathematics, Vol. 42. Springer-Verlag, New York-Heidelberg, 1977. Translated from the second French edition by Leonard L. Scott.

[47] Nir Sharon, Joe Kileel, Yuehaw Khoo, Boris Landa, and Amit Singer. Method of moments for $3 \mathrm{D}$ single particle ab initio modeling with non-uniform distribution of viewing angles. Inverse Problems, 36(4):044003, 2020.

[48] Fred J Sigworth. A maximum-likelihood approach to single-particle image refinement. Journal of structural biology, 122(3):328-339, 1998.

[49] Amit Singer. Angular synchronization by eigenvectors and semidefinite programming. Applied and computational harmonic analysis, 30(1):20-36, 2011.

[50] Amit Singer and Yoel Shkolnisky. Three-dimensional structure determination from common lines in cryo-EM by eigenvectors and semidefinite programming. SIAM journal on imaging sciences, 4(2):543-572, 2011.

[51] Yong Zi Tan, Philip R Baldwin, Joseph H Davis, James R Williamson, Clinton S Potter, Bridget Carragher, and Dmitry Lyumkis. Addressing preferred specimen orientation in singleparticle cryo-EM through tilting. Nature methods, 14(8):793-796, 2017.

[52] Roberto Tron, Xiaowei Zhou, and Kostas Daniilidis. A survey on rotation optimization in structure from motion. In Proceedings of the IEEE Conference on Computer Vision and Pattern Recognition Workshops, pages 77-85, 2016. 